PROCEEDINGS OF THE

AMERICAN MATHEMATICAL SOCIETY

Volume 126, Number 2, February 1998, Pages 471-479

S $0002-9939(98) 04098-2$

\title{
CARDINAL INVARIANTS CONCERNING BOUNDED FAMILIES OF EXTENDABLE AND ALMOST CONTINUOUS FUNCTIONS
}

\author{
KRZYSZTOF CIESIELSKI AND ALEKSANDER MALISZEWSKI
}

(Communicated by J. Marshall Ash)

\begin{abstract}
In this paper we introduce and examine a cardinal invariant $A_{b}$ closely connected to the addition of bounded functions from $\mathbb{R}$ to $\mathbb{R}$. It is analogous to the invariant A defined earlier for arbitrary functions by T. Natkaniec. In particular, it is proved that each bounded function can be written as the sum of two bounded almost continuous functions, and an example is given that there is a bounded function which cannot be expressed as the sum of two bounded extendable functions.
\end{abstract}

\section{Preliminaries}

We will use the following terminology and notation. The letters $\mathbb{N}, \mathbb{Q}$, and $\mathbb{R}$ denote the set of positive integers, the set of rationals, and the real line, respectively. Functions will be identified with their graphs. The family of all functions from a set $X$ into $Y$ will be denoted by $Y^{X}$. The symbol $|X|$ will stand for the cardinality of a set $X$. We consider cardinals as ordinals not in one-to-one correspondence with the smaller ordinals. For a cardinal number $\kappa$ we will write $\operatorname{cof}(\kappa)$ for the cofinality of $\kappa$. A cardinal number $\kappa$ is called regular, if $\kappa=\operatorname{cof}(\kappa)$. We define $\mathfrak{c}=|\mathbb{R}|$ and $\omega=|\mathbb{N}|$. For a set $A \subseteq \mathbb{R}$ its characteristic function is denoted by $\chi_{A}$. The projection of a set $A \subseteq \mathbb{R}^{2}$ onto the $x$-axis will be denoted by $\operatorname{dom} A$. For a cardinal number $\kappa \leq \mathfrak{c}$ and an open set $U \subseteq \mathbb{R}^{n}$ we say that $X \subseteq U$ is $\kappa$-dense in $U$ if $|X \cap V| \geq \kappa$ for every nonempty open set $V \subseteq U$.

The symbols $\mathrm{Bd}$ and $\mathrm{BD}$ will denote the class of all bounded functions from $\mathbb{R}$ to $\mathbb{R}$ and the class of all families $\mathfrak{A} \subseteq \mathbb{R}^{\mathbb{R}}$ of functions having common bound, respectively. For $\mathcal{F} \subseteq \mathbb{R}^{\mathbb{R}}$ we define two cardinal invariants (cf. [12] and [6]):

$$
\begin{aligned}
\mathrm{A}(\mathcal{F}) & =\min \left\{|\mathfrak{A}|: \mathfrak{A} \subseteq \mathbb{R}^{\mathbb{R}} \& \neg\left(\exists g \in \mathbb{R}^{\mathbb{R}}\right)(\forall f \in \mathfrak{A})(f+g \in \mathcal{F})\right\} \cup\left\{\left(2^{\mathfrak{c}}\right)^{+}\right\} \\
& =\min \left\{|\mathfrak{A}|: \mathfrak{A} \subseteq \mathbb{R}^{\mathbb{R}} \&\left(\forall g \in \mathbb{R}^{\mathbb{R}}\right)(\exists f \in \mathfrak{A})(f+g \notin \mathcal{F})\right\} \cup\left\{\left(2^{\mathfrak{c}}\right)^{+}\right\},
\end{aligned}
$$

and

$$
\begin{aligned}
\mathrm{A}_{\mathrm{b}}(\mathcal{F}) & =\min \{|\mathfrak{A}|: \mathfrak{A} \in \mathrm{BD} \quad \& \neg(\exists g \in \mathrm{Bd})(\forall f \in \mathfrak{A})(f+g \in \mathcal{F})\} \cup\left\{\left(2^{\mathfrak{c}}\right)^{+}\right\} \\
& =\min \{|\mathfrak{A}|: \mathfrak{A} \in \mathrm{BD} \&(\forall g \in \mathrm{Bd})(\exists f \in \mathfrak{A})(f+g \notin \mathcal{F})\} \cup\left\{\left(2^{\mathfrak{c}}\right)^{+}\right\} .
\end{aligned}
$$

Received by the editors March 28, 1996 and, in revised form, August 11, 1996.

1991 Mathematics Subject Classification. Primary 26A21; Secondary 54C08.

Key words and phrases. Peripheral continuity, almost continuity, connectivity, extendability.

This work was partially supported by NSF Cooperative Research Grant INT-9600548.

(C)1998 American Mathematical Society 
The next proposition lists several basic properties of the function $A_{b}$. The similar properties for the function A can be found in [6]. They will be left without proofs. (Their easy proofs are analogous to those of Propositions 1.1 and 1.3 of [6].)

Proposition 1.1. Let $\mathcal{F} \subseteq \mathbb{R}^{\mathbb{R}}$. Then

(1): $\mathrm{A}_{\mathrm{b}}(\mathcal{F})=\mathrm{A}_{\mathrm{b}}(\mathcal{F} \cap \mathrm{Bd})$;

(2): $\mathrm{A}_{\mathrm{b}}(\mathcal{F}) \geq 1$

(3): if $\mathcal{F} \subseteq \overline{\mathcal{G}} \subseteq \mathbb{R}^{\mathbb{R}}$, then $\mathrm{A}_{\mathrm{b}}(\mathcal{F}) \leq \mathrm{A}_{\mathrm{b}}(\mathcal{G})$;

(4): $\mathrm{A}_{\mathrm{b}}(\mathcal{F}) \leq 2$ if and only if $\left\{f_{1}-f_{2}: f_{1}, f_{2} \in \mathcal{F} \cap \mathrm{Bd}\right\} \neq \mathrm{Bd}$;

(5): $\mathrm{A}_{\mathrm{b}}(\mathcal{F}) \geq 2$ if and only if $\mathcal{F} \cap \mathrm{Bd} \neq \emptyset$;

(6): if $\mathcal{F} \supseteq \mathrm{Bd}$, then $\mathrm{A}_{\mathrm{b}}(\mathcal{F})=\left(2^{\mathfrak{c}}\right)^{+}$.

We will find the value of the function $A_{b}$ for the following classes of functions, where $\kappa$ is a cardinal number $\leq \mathfrak{c}$ and $X$ is an arbitrary topological space.

$\mathrm{PC}_{\kappa}$ : of all functions $f: \mathbb{R} \rightarrow \mathbb{R}$ with the following property: for every $x \in \mathbb{R}$ and every $\varepsilon>0$ we have $|f \cap[(x-\varepsilon, x) \times(f(x)-\varepsilon, f(x)+\varepsilon)]| \geq \kappa$ and $|f \cap[(x, x+\varepsilon) \times(f(x)-\varepsilon, f(x)+\varepsilon)]| \geq \kappa$. In particular, $\mathrm{PC}_{\omega}$ is the class of peripherally continuous functions.

D: of all Darboux functions $f: \mathbb{R} \rightarrow \mathbb{R}$, i.e., such that $f[J]$ is an interval for every interval $J \subseteq \mathbb{R}$.

$\mathcal{U}_{\kappa}$ : of all functions $f: \mathbb{R} \rightarrow \mathbb{R}$ fulfilling the following condition: for all $a<b$ and each set $A \subseteq[a, b]$ with $|A|<\kappa+1$, the set $f[(a, b) \backslash A]$ is dense in the interval $(\min \{f(a), f(b)\}, \max \{f(a), f(b)\})$. In particular, $\mathcal{U}_{\mathfrak{c}}$ is the uniform closure of the class D [2], while the class $\mathcal{U}_{0}$ was examined earlier by T. Radakovič [14] and H. W. Ellis [8]. Moreover $\mathcal{U}_{\mathfrak{c}}=\mathrm{PC}_{\mathfrak{c}} \cap \mathcal{U}_{0}$ [2, Theorem 3.2].

$\mathrm{AC}:$ of all almost continuous functions $f: \mathbb{R} \rightarrow \mathbb{R}$, i.e., such that for every open set $U \subseteq \mathbb{R}^{2}$ containing $f$ there is a continuous function $h: \mathbb{R} \rightarrow \mathbb{R}$ with $h \subseteq U$.

$\operatorname{Conn}(X)$ : of all connectivity functions $f: X \rightarrow \mathbb{R}$, i.e., such that the restriction $f \uparrow C$ (that is $f \cap[C \times \mathbb{R}])$ is connected in $X \times \mathbb{R}$ whenever $C \subseteq X$ is connected. ${ }^{1}$

Ext: of all extendable functions $f: \mathbb{R} \rightarrow \mathbb{R}$, i.e., such that there exists a function $g \in \operatorname{Conn}(\mathbb{R} \times[0,1])$ with $f(x)=g(x, 0)$ for every $x \in \mathbb{R}$.

PR: of all functions $f: \mathbb{R} \rightarrow \mathbb{R}$ with perfect road, i.e., such that for every $x \in \mathbb{R}$ there exists a perfect set $P \subseteq \mathbb{R}$ having $x$ as a bilateral limit point for which the restriction $f \uparrow P$ is continuous at $x$.

For the above classes of functions we have the following proper inclusions, marked by arrows.

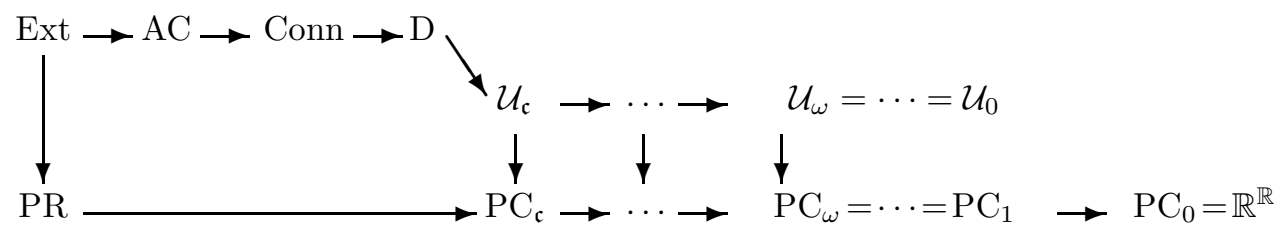

For the upper row inclusions see, e.g., [1]. The inclusion Ext $\varsubsetneqq \mathrm{PR}$ follows from [9]. The other relations are more or less evident.

\footnotetext{
${ }^{1}$ Actually we will study only the class Conn $=\operatorname{Conn}(\mathbb{R})$, but we need the class $\operatorname{Conn}(\mathbb{R} \times[0,1])$ to define the class Ext.
} 
The values of the function A for most of these classes have been established in several papers, as quoted below.

Theorem $1.2([6]) . \mathrm{A}\left(\mathrm{PC}_{\omega}\right)=2^{\mathfrak{c}}$ and $\mathrm{A}(\mathrm{Ext})=\mathrm{A}(\mathrm{PR})=\mathfrak{c}^{+}$.

Theorem $1.3([5]) \cdot \mathfrak{c}^{+} \leq \mathrm{A}(\mathrm{AC})=\mathrm{A}(\mathrm{Conn})=\mathrm{A}(\mathrm{D}) \leq 2^{\mathfrak{c}}, \operatorname{cof}(\mathrm{A}(\mathrm{D}))>\mathfrak{c}$, and it is pretty much all that can be shown in ZFC. More precisely, the assertion $\mathrm{A}(\mathrm{D})=2^{\mathfrak{c}}$ is independent of the cofinality of $2^{\mathfrak{c}}$, and for each regular cardinal $\lambda$ between $\mathfrak{c}^{+}$ and $2^{\mathfrak{c}}$ it is consistent with $Z F C$ that $\mathrm{A}(\mathrm{D})=\lambda$.

The values of the function A for the other classes have not been considered so far. However, it is not difficult to find them.

Theorem 1.4. $\mathrm{A}\left(\mathrm{PC}_{0}\right)=\left(2^{\mathfrak{c}}\right)^{+}$and $\mathrm{A}\left(\mathcal{U}_{\kappa}\right)=\mathrm{A}\left(\mathrm{PC}_{\kappa}\right)=2^{\mathfrak{c}}$ for every infinite cardinal number $\kappa \leq \mathfrak{c}$.

The equality $\mathrm{A}\left(\mathrm{PC}_{0}\right)=\left(2^{\mathfrak{c}}\right)^{+}$is obvious. The other part will be proved in the next section.

It follows from Proposition 1.1(6) that $\mathrm{A}_{\mathrm{b}}\left(\mathrm{PC}_{0}\right)=\left(2^{\mathfrak{c}}\right)^{+}$. It has been proved in [11] that $A_{b}(D)=\operatorname{cof}(\mathfrak{c})$. The goal of this paper is to establish the values of $A_{b}$ for all other classes by proving the following theorem.

Theorem 1.5. (1): $A_{b}(E x t)=A_{b}(P R)=2$.

(2): $\mathrm{A}_{\mathrm{b}}(\mathrm{AC})=\mathrm{A}_{\mathrm{b}}(\mathrm{Conn})=\mathrm{A}_{\mathrm{b}}(\mathrm{D})=\mathrm{A}_{\mathrm{b}}\left(\mathcal{U}_{\mathfrak{c}}\right)=\mathrm{A}_{\mathrm{b}}\left(\mathrm{PC}_{\mathfrak{c}}\right)=\operatorname{cof}(\mathfrak{c})$.

(3): $\mathrm{A}_{\mathrm{b}}\left(\mathcal{U}_{\kappa}\right)=\mathrm{A}_{\mathrm{b}}\left(\mathrm{PC}_{\kappa}\right)=\mathfrak{c}$ for every infinite cardinal number $\kappa<\mathfrak{c}$.

In particular, the equality $\mathrm{A}_{\mathrm{b}}(\mathrm{AC})=\operatorname{cof}(\mathfrak{c})$ solves Problem 2 of [10]. (This problem was restated in [11, p. 679].) Notice also that Theorem 1.5 and Proposition 1.1(4) imply immediately the following corollaries.

Corollary 1.6. Every bounded function $f: \mathbb{R} \rightarrow \mathbb{R}$ is the sum of two bounded almost continuous functions.

Corollary 1.7. There exists a bounded function $f: \mathbb{R} \rightarrow \mathbb{R}$ which is not the sum of two bounded functions with perfect road.

Notice that Corollary 1.6 generalizes the theorem of U. B. Darji and P. D. Humke that every bounded function is the sum of three bounded almost continuous functions [7]. On the other hand, by Corollary 1.7, T. Natkaniec's result asserting that every bounded function is the sum of three bounded extendable functions [13, Theorem 1] cannot be improved.

In what follows we will use a generalization of [4, Lemma 4, p. 285] and [3, Lemma 1]. We will need the following notation. For every $\lambda>\omega$ and every function $f: \mathbb{R} \rightarrow \mathbb{R}$ let

$$
\begin{aligned}
& A_{\lambda}^{-}(f)=\{x \in \mathbb{R}:(\exists \varepsilon>0)(|f \cap[(x-\varepsilon, x) \times(f(x)-\varepsilon, f(x)+\varepsilon)]|<\lambda)\}, \\
& A_{\lambda}^{+}(f)=\{x \in \mathbb{R}:(\exists \varepsilon>0)(|f \cap[(x, x+\varepsilon) \times(f(x)-\varepsilon, f(x)+\varepsilon)]|<\lambda)\},
\end{aligned}
$$

and $A_{\lambda}(f)=A_{\lambda}^{-}(f) \cup A_{\lambda}^{+}(f)$.

Lemma 1.8. If $\lambda$ is a cardinal number with $\operatorname{cof}(\lambda)>\omega$, then $\left|A_{\lambda}(f)\right|<\lambda$ for every function $f: \mathbb{R} \rightarrow \mathbb{R}$.

Proof. Assume, by way of contradiction, that $\left|A_{\lambda}(f)\right| \geq \lambda$. Suppose that $\left|A_{\lambda}^{-}(f)\right| \geq$ $\lambda$, the case $\left|A_{\lambda}^{+}(f)\right| \geq \lambda$ being similar. Since $\operatorname{cof}(\lambda)>\omega$ and

$$
A_{\lambda}^{-}(f)=\bigcup_{n \in \mathbb{N}}\left\{x \in \mathbb{R}:\left|f \cap\left[\left(x-n^{-1}, x\right) \times\left(f(x)-n^{-1}, f(x)+n^{-1}\right)\right]\right|<\lambda\right\},
$$


there exist an $\varepsilon>0$ and a subset $B$ of $A_{\lambda}^{-}(f)$ of cardinality $\geq \lambda$ such that $|f \cap[(x-\varepsilon, x) \times(f(x)-\varepsilon, f(x)+\varepsilon)]|<\lambda$ for every $x \in B$. Choose an open interval $J$ of length $\varepsilon$ such that the set $C=\{x \in B: f(x) \in J\}$ has cardinality greater than or equal to $\lambda$. Then, there is an $x_{0} \in C$ with $\left|\left(x_{0}-\varepsilon / 2, x_{0}\right) \cap C\right| \geq \lambda$. But $f\left(x_{0}\right) \in J$, so $J \subseteq\left(f\left(x_{0}\right)-\varepsilon, f\left(x_{0}\right)+\varepsilon\right)$ and $\left|f \cap\left[\left(x_{0}-\varepsilon, x_{0}\right) \times\left(f\left(x_{0}\right)-\varepsilon, f\left(x_{0}\right)+\varepsilon\right)\right]\right| \geq \lambda$, contradicting the fact that $x_{0} \in B$.

\section{Proof of Theorem 1.4}

Let $\kappa \leq \mathfrak{c}$ be an infinite cardinal. It follows from the monotonicity of the function $\mathrm{A}$ and Theorem 1.2 that $\mathrm{A}\left(\mathcal{U}_{\mathfrak{c}}\right) \leq \mathrm{A}\left(\mathcal{U}_{\kappa}\right) \leq \mathrm{A}\left(\mathrm{PC}_{\kappa}\right) \leq \mathrm{A}\left(\mathrm{PC}_{\omega}\right)=2^{\mathfrak{c}}$. Thus, it is enough to show that $\mathrm{A}\left(\mathcal{U}_{\mathfrak{c}}\right) \geq 2^{\mathfrak{c}}$. This can be established by the following minor modification of the proof of [6, Theorem 1.7(3)].

Let $\mathcal{F} \subseteq \mathbb{R}^{\mathbb{R}}$ be such that $|\mathcal{F}|<2^{\mathfrak{c}}$. We will find a function $g: \mathbb{R} \rightarrow \mathbb{R}$ such that $f+g \in \mathcal{U}_{\mathfrak{c}}$ for every $f \in \mathcal{F}$.

Let $\mathfrak{G}$ be the family of all pairs $\langle I, J\rangle$, where $I$ and $J$ are nonempty open intervals with rational end points. Let $\left\{\left\langle I_{\xi}, J_{\xi}\right\rangle: \xi<\mathfrak{c}\right\}$ be an enumeration of $\mathfrak{G}$ with each pair appearing $\mathfrak{c}$ many times. For each $\xi<\mathfrak{c}$ define a set $B_{\xi} \subseteq I_{\xi}$ of cardinality $\mathfrak{c}$ such that $B_{\xi} \cap B_{\eta}=\emptyset$ for any $\xi<\eta<\mathfrak{c}$. (See, e.g., [11, Lemma 5].)

Next, fix a $\xi<\mathfrak{c}$. For each $f \in \mathcal{F}$ construct a function $h_{\xi, f}: B_{\xi} \rightarrow \mathbb{Q}$ such that

$$
f(x)+h_{\xi, f}(x) \in J_{\xi} \quad \text { for every } x \in B_{\xi} .
$$

Then, by [6, Lemma 2.2] (used with $B=B_{\xi}$ and $H=\left\{h_{\xi, f}: f \in \mathcal{F}\right\}$ ), there exists a function $g_{\xi}: B_{\xi} \rightarrow \mathbb{Q}$ such that $h_{\xi, f} \cap g_{\xi} \neq \emptyset$ for every $f \in \mathcal{F}$.

Let $g: \mathbb{R} \rightarrow \mathbb{Q}$ be a common extension of all functions $g_{\xi}$. Then for every $\xi<\mathfrak{c}$ and every $f \in \mathcal{F}$ there exists an $x \in B_{\xi}$ such that $f(x)+g(x) \in J_{\xi}$. So, for every $f \in \mathcal{F}$ the graph of $f+g$ is $\mathfrak{c}$-dense in $\mathbb{R}^{2}$. Thus, $f+g \in \mathcal{U}_{\mathfrak{c}}$.

\section{Proof of Theorem 1.5(1)}

By Proposition 1.1(5) and (3), we have $2 \leq A_{b}($ Ext $) \leq A_{b}(P R)$. Thus, it is enough to prove that $\mathrm{A}_{\mathrm{b}}(\mathrm{PR}) \leq 2$, i.e., by Proposition 1.1(4), that $\left\{f_{1}-f_{2}: f_{1}, f_{2} \in\right.$ $\mathrm{PR} \cap \mathrm{Bd}\} \neq \mathrm{Bd}$. This follows immediately from the next example.

Example 3.1. Let $B \subseteq \mathbb{R}$ be a Bernstein set and $f=\chi_{B}-\chi_{\mathbb{R} \backslash B}$. If $f=g_{0}-g_{1}$ and $g_{0}, g_{1} \in \mathrm{PR}$, then both $g_{0}$ and $g_{1}$ are bilaterally unbounded.

Proof. By way of contradiction assume that there are $g_{0}, g_{1} \in \mathrm{PR}$ such that $f=g_{0}-$ $g_{1}$ and $g_{0}$ is not bilaterally unbounded. We will assume that $m_{0}=\inf g_{0}[\mathbb{R}]>-\infty$. (The other case is analogous.) Put $m_{1}=\inf g_{1}[\mathbb{R}]$ and notice that $m_{1} \geq m_{0}-1>$ $-\infty$.

For $i<2$ choose a nonempty perfect set $P_{i}$ such that $g_{i}<m_{i}+2^{-1}$ on $P_{i}$. Then

$$
m_{0} \leq \inf g_{0}\left[P_{1}\right]=\inf \left(g_{1}+f\right)\left[P_{1}\right] \leq\left(m_{1}+2^{-1}-1\right)=m_{1}-2^{-1}<m_{1}
$$

and

$$
m_{1} \leq \inf g_{1}\left[P_{0}\right]=\inf \left(g_{0}-f\right)\left[P_{0}\right] \leq\left(m_{0}+2^{-1}-1\right)=m_{0}-2^{-1}<m_{0},
$$

an impossibility. This completes the proof. 


\section{Proof of Theorem $1.5(2)$}

By Proposition 1.1(3), $\mathrm{A}_{\mathrm{b}}(\mathrm{AC}) \leq \mathrm{A}_{\mathrm{b}}(\mathrm{Conn}) \leq \mathrm{A}_{\mathrm{b}}(\mathrm{D}) \leq \mathrm{A}_{\mathrm{b}}\left(\mathcal{U}_{\mathfrak{c}}\right) \leq \mathrm{A}_{\mathrm{b}}\left(\mathrm{PC}_{\mathfrak{c}}\right)$. So, it is enough to prove that $A_{b}\left(P_{c}\right) \leq \operatorname{cof}(\mathfrak{c})$ and $A_{b}(A C) \geq \operatorname{cof}(\mathfrak{c})$. The first of these inequalities follows from the next example, and the second one follows immediately from Theorem 4.1.

Example 4.1. There is a family $\mathfrak{A} \in \mathrm{BD}$ with $|\mathfrak{A}|=\operatorname{cof}(\mathfrak{c})$ such that for every function $g$ bounded above we have $f+g \notin \mathrm{PC}_{\mathfrak{c}}$ for some $f \in \mathfrak{A}$.

Proof. Let $\mathcal{A}$ be a family of sets such that $|\mathcal{A}|=\operatorname{cof}(\mathfrak{c}),|A|<\mathfrak{c}$ for every $A \in \mathcal{A}$, and $|\cup \mathcal{A}|=\mathfrak{c}$. Clearly we may assume that the sets in $\mathcal{A}$ are nonempty, pairwise disjoint, and that $\bigcup \mathcal{A}=\mathbb{R}$. Put $\mathfrak{A}=\left\{\chi_{A}: A \in \mathcal{A}\right\}$.

Let $g: \mathbb{R} \rightarrow \mathbb{R}$ be bounded above. Define $M=\sup g[\mathbb{R}]$ and choose an $x \in \mathbb{R}$ with $g(x)>M-1$. Then $x \in A$ for some $A \in \mathcal{A}$. Consequently, $\left(g+\chi_{A}\right)(t)=g(t) \leq M$ for every $t \notin A$ and $\left(g+\chi_{A}\right)(x)>M$. Thus

$$
1 \leq\left|\left\{t \in \mathbb{R}:\left(g+\chi_{A}\right)(t)>M\right\}\right| \leq|A|<\mathfrak{c}
$$

and $g+\chi_{A} \notin \mathrm{PC}_{\mathfrak{c}}$.

Theorem 4.1. If $\mathfrak{A} \subseteq(-1,1)^{\mathbb{R}}$ and $|\mathfrak{A}|<\operatorname{cof}(\mathfrak{c})$, then there is a function $g \in$ $(-2,2)^{\mathbb{R}}$ such that $f+g \in \mathrm{AC}$ for every $f \in \mathfrak{A}$.

Proof. For each $f \in \mathfrak{A}$ let

$$
\begin{aligned}
U_{f} & =\{\langle x, y\rangle: x \in \mathbb{R} \& f(x)-2<y<f(x)+2\} \\
& =\bigcup_{x \in \mathbb{R}}[\{x\} \times(f(x)-2, f(x)+2)]
\end{aligned}
$$

and

$$
\mathcal{K}_{f}=\left\{K \subseteq \mathbb{R}^{2}: K \text { is closed \& }\left|\operatorname{dom}\left(K \cap U_{f}\right)\right|=\mathfrak{c}\right\} .
$$

Clearly, $\left|\mathcal{K}_{f}\right|=\mathfrak{c}$. Also, by Lemma $1.8,\left|A_{\mathfrak{c}}(f)\right|<\mathfrak{c}$ for every $f \in \mathfrak{A}$. So, since $|\mathfrak{A}|<\operatorname{cof}(\mathfrak{c})$, we obtain

$$
\text { the set } A=\bigcup_{f \in \mathfrak{A}} A_{\mathfrak{c}}(f) \text { has cardinality less than } \mathfrak{c} \text {. }
$$

Let $\left\{\left\langle f_{\xi}, K_{\xi}\right\rangle: \xi<\mathfrak{c}\right\}$ be a transfinite sequence consisting of all pairs $\langle f, K\rangle$ such that $f \in \mathfrak{A}$ and $K \in \mathcal{K}_{f}$. We will construct by transfinite induction a sequence $\left\{\left\langle x_{\xi}, y_{\xi}\right\rangle: \xi<\mathfrak{c}\right\}$ such that for every $\xi<\mathfrak{c}$ the following conditions hold:

(i) $x_{\xi} \notin\left\{x_{\zeta}: \zeta<\xi\right\} \cup A$;

(ii) $\left\langle x_{\xi}, f_{\xi}\left(x_{\xi}\right)+y_{\xi}\right\rangle \in K_{\xi} \cap U_{f_{\xi}}$.

So, assume that $\left\{\left\langle x_{\zeta}, y_{\zeta}\right\rangle: \zeta<\xi\right\}$ have been already defined for some $\xi<\mathfrak{c}$. By the definition of $\mathcal{K}_{f_{\xi}}$ and condition (1), we can choose

$$
x_{\xi} \in \operatorname{dom}\left(K_{\xi} \cap U_{f_{\xi}}\right) \backslash\left(\left\{x_{\zeta}: \zeta<\xi\right\} \cup A\right) .
$$

Let $z_{\xi}$ be such that $\left\langle x_{\xi}, z_{\xi}\right\rangle \in K_{\xi} \cap U_{f_{\xi}}$ and put $y_{\xi}=z_{\xi}-f_{\xi}\left(x_{\xi}\right)$. Then (i) and (ii) are obviously satisfied. This completes the inductive construction.

Notice that $y_{\xi} \in(-2,2)$ for every $\xi<\mathfrak{c}$ since $z_{\xi} \in\left(f_{\xi}\left(x_{\xi}\right)-2, f_{\xi}\left(x_{\xi}\right)+2\right)$. Define the function $g: \mathbb{R} \rightarrow(-2,2)$ by

$$
g(x)= \begin{cases}y_{\xi} & \text { if } x=x_{\xi} \text { for some } \xi<\mathfrak{c} \\ 0 & \text { otherwise }\end{cases}
$$


Fix an $f \in \mathfrak{A}$. We will prove that $f+g \in \mathrm{AC}$. Let $V \subseteq \mathbb{R}^{2}$ be an open set containing $f+g$. First notice that if

$$
E=\operatorname{dom}\left(U_{f} \backslash V\right)=\{x \in \mathbb{R}:[\{x\} \times(f(x)-2, f(x)+2)] \nsubseteq V\},
$$

then $|E|<\mathfrak{c}$. Indeed, otherwise $K=\mathbb{R}^{2} \backslash V \in \mathcal{K}_{f}$, so there exists a $\xi<\mathfrak{c}$ with $\left\langle f_{\xi}, K_{\xi}\right\rangle=\langle f, K\rangle$. By (ii), we obtain

$$
\left\langle x_{\xi},(f+g)\left(x_{\xi}\right)\right\rangle=\left\langle x_{\xi}, f_{\xi}\left(x_{\xi}\right)+y_{\xi}\right\rangle \in K_{\xi}=K
$$

contradicting the fact that $f+g \subseteq V$.

Define

$$
F=\operatorname{dom}([\mathbb{R} \times[-1,1]] \backslash V)=\{x \in \mathbb{R}:[\{x\} \times[-1,1]] \nsubseteq V\} .
$$

Since $[-1,1] \subseteq(f(x)-2, f(x)+2)$ for every $x \in \mathbb{R}$, so $[\mathbb{R} \times[-1,1]] \subseteq U_{f}$. Hence $F \subseteq E$ and $|F|<\mathfrak{c}$. But $[\mathbb{R} \times[-1,1]] \backslash V$ is a closed subset of $\mathbb{R} \times[-1,1]$. Thus $F$ is closed in $\mathbb{R}$ and it is at most countable.

Let $\mathcal{J}$ be the family of all compact intervals $J=[a, b]$ for which there exists a continuous function $h: J \rightarrow \mathbb{R}$ with $h \subseteq V$ and $h(a)=h(b)=0$. Moreover let $G$ be the set of all $x \in \mathbb{R}$ for which there exists a $\delta_{x}>0$ such that $[a, b] \in \mathcal{J}$ whenever $a, b \in\left(x-\delta_{x}, x+\delta_{x}\right) \backslash F$ and $a<b$. The first claim is obvious.

Claim 1. If $\left[a_{0}, a_{1}\right] \in \mathcal{J}$ and $\left[a_{1}, a_{2}\right] \in \mathcal{J}$, then $\left[a_{0}, a_{2}\right] \in \mathcal{J}$.

Claim 2. If $J=[a, b] \subseteq G$ and $a, b \notin F$, then $J \in \mathcal{J}$.

Indeed, the compactness of $J$ and the relation $J \subseteq \bigcup_{x \in J}\left(x-\delta_{x}, x+\delta_{x}\right)$ imply that there exist $x_{0}, \ldots, x_{p} \in J$ such that $J \subseteq \bigcup_{i=0}^{p}\left(x_{i}-\delta_{x_{i}}, x_{i}+\delta_{x_{i}}\right)$. Hence we can find nonoverlapping compact intervals $J_{0}, \ldots, J_{l} \in \mathcal{J}$ with $J=\bigcup_{j=0}^{l} J_{j}$. By Claim 1, we obtain $J \in \mathcal{J}$.

Claim 3. We have $G=\mathbb{R}$.

First notice that $G$ is open and that $\mathbb{R} \backslash F \subseteq G$. By way of contradiction suppose that the set $P=\mathbb{R} \backslash G \subseteq F$ is nonempty. Thus, $P$ is scattered and so, it contains an isolated point $s$. Let $\delta>0$ be such that $[s-\delta, s+\delta] \cap P=\{s\}$. To get a contradiction it is enough to show that $s \in G$.

Let $a, b \in(s-\delta, s+\delta) \backslash F$ and $a<b$. If $a>s$ or $b<s$, then, by Claim 2, we obtain $[a, b] \in \mathcal{J}$. So we may assume that $a<s<b$. Let $\varepsilon>0$ be such that $a<s-\varepsilon<s+\varepsilon<b$ and

$$
R=[(s-\varepsilon, s+\varepsilon) \times((f+g)(s)-\varepsilon,(f+g)(s)+\varepsilon)] \subseteq V .
$$

(Such an $\varepsilon$ exists, since $V$ is open and $\langle s,(f+g)(s)\rangle \in V$.)

Notice that there exist $\left\langle q_{0}, y_{0}\right\rangle,\left\langle q_{1}, y_{1}\right\rangle \in U_{f} \cap R$ such that $q_{0} \in(s-\varepsilon, s) \backslash E$ and $q_{1} \in(s, s+\varepsilon) \backslash E$. We will prove it for $i=0$, the other case being similar.

First suppose $s \in A$. Take an arbitrary $q_{0} \in(s-\varepsilon, s) \backslash E$. Then $g(s)=0$, so $(f+g)(s)=f(s) \in(-1,1) \subseteq\left(f\left(q_{0}\right)-2, f\left(q_{0}\right)+2\right)$. Consequently, the interval

$$
D=\left(f\left(q_{0}\right)-2, f\left(q_{0}\right)+2\right) \cap((f+g)(s)-\varepsilon,(f+g)(s)+\varepsilon)
$$

is nonempty. So every point $\left\langle q_{0}, y_{0}\right\rangle$ with $y_{0} \in D$ has the required properties.

Otherwise by the definition of $A_{\mathfrak{c}}^{-}(f)$, there is a $q_{0} \in(s-\varepsilon, s) \backslash E$ with $f\left(q_{0}\right) \in$ $(f(s)-\varepsilon, f(s)+\varepsilon)$. Then $\left|f(s)-f\left(q_{0}\right)\right|<\varepsilon$ and

$$
\left|(f+g)(s)-f\left(q_{0}\right)\right| \leq|g(s)|+\left|f(s)-f\left(q_{0}\right)\right|<2+\varepsilon .
$$


Define the set $D$ as above. Observe that $D \neq \emptyset$ and every point $\left\langle q_{0}, y_{0}\right\rangle$ with $y_{0} \in D$ has the required properties.

Fix an $i<2$. Put $c_{i}=\min \left\{y_{i},-1\right\}$ and $d_{i}=\max \left\{y_{i}, 1\right\}$. Since $q_{i} \notin E$, we see that $\left[\left\{q_{i}\right\} \times\left[c_{i}, d_{i}\right]\right] \subseteq\left[\left\{q_{i}\right\} \times\left(f\left(q_{i}\right)-2, f\left(q_{i}\right)+2\right)\right] \subseteq V$. Since the set $\left\{q_{i}\right\} \times\left[c_{i}, d_{i}\right]$ is compact, we can find $a_{i}, b_{i} \in(s-\varepsilon, s+\varepsilon) \backslash E$ such that $q_{i} \in\left(a_{i}, b_{i}\right)$ and

$$
R_{i}=\left[\left(a_{i}, b_{i}\right) \times\left[c_{i}, d_{i}\right]\right] \subseteq V .
$$

Construct continuous functions $h_{0}:\left[a, a_{0}\right] \rightarrow \mathbb{R}$ and $h_{1}:\left[b_{1}, b\right] \rightarrow \mathbb{R}$ such that $h_{0} \cup h_{1} \subseteq V$ and $h_{0}(a)=h_{0}\left(a_{0}\right)=h_{1}\left(b_{1}\right)=h_{1}(b)=0$. (We use Claim 2.) Extend $h_{0} \cup h_{1}$ to $h:[a, b] \rightarrow \mathbb{R}$ by connecting the following pairs of points by straight line segments: $\left\langle a_{0}, 0\right\rangle$ with $\left\langle q_{0}, y_{0}\right\rangle,\left\langle q_{0}, y_{0}\right\rangle$ with $\left\langle q_{1}, y_{1}\right\rangle$, and $\left\langle q_{1}, y_{1}\right\rangle$ with $\left\langle b_{1}, 0\right\rangle$. Notice that these segments are contained in rectangles $R_{0}, R$, and $R_{1}$, respectively. Thus $h \subseteq V$. Clearly $h(a)=h(b)=0$ and $h$ is continuous, so $[a, b] \in \mathcal{J}$. Hence $s \in G$, an impossibility. Claim 3 has been proved.

Using Claims $1-3$ it is easy to show that there exists a continuous function $h: \mathbb{R} \rightarrow \mathbb{R}$ with $h \subseteq V$. So $f+g \in \mathrm{AC}$.

\section{Proof of Theorem 1.5(3)}

Let $\kappa<\mathfrak{c}$ be an infinite cardinal number. By Proposition 1.1(3), we have $\mathrm{A}_{\mathrm{b}}\left(\mathcal{U}_{\kappa}\right) \leq \mathrm{A}_{\mathrm{b}}\left(\mathrm{PC}_{\kappa}\right) \leq \mathrm{A}_{\mathrm{b}}\left(\mathrm{PC}_{\omega}\right)$. So, it is enough to prove that $\mathrm{A}_{\mathrm{b}}\left(\mathrm{PC}_{\omega}\right) \leq \mathfrak{c}$ and $\mathrm{A}_{\mathrm{b}}\left(\mathcal{U}_{\kappa}\right) \geq \mathfrak{c}$. The first of these inequalities follows from Proposition 5.1, and the second one from Theorem 5.2.

Proposition 5.1. If a function $g$ is bounded above, then $g+\chi_{\{x\}} \notin \mathrm{PC}_{\omega}$ for some $x \in \mathbb{R}$.

Proof. Define $M=\sup g[\mathbb{R}]$ and choose an $x \in \mathbb{R}$ with $g(x)>M-1$. Then $\left(g+\chi_{\{x\}}\right)(t)=g(t) \leq M$ for $t \neq x$ and $\left(g+\chi_{\{x\}}\right)(x)>M$. Thus

$$
\left|\left\{t \in \mathbb{R}:\left(g+\chi_{\{x\}}\right)(t)>M\right\}\right|=1
$$

and $g+\chi_{\{x\}} \notin \mathrm{PC}_{\omega}$.

Theorem 5.2. If $\kappa<\mathfrak{c}, \mathfrak{A} \subseteq(-1,1)^{\mathbb{R}}$, and $|\mathfrak{A}|<\mathfrak{c}$, then there is a function $g \in(-2,2)^{\mathbb{R}}$ such that $f+g \in \mathcal{U}_{\kappa}$ for each $f \in \mathfrak{A}$.

Proof. The proof is a modification and simplification of that of Theorem 4.1.

Without loss of generality we may assume that $\kappa \geq|\mathfrak{A}|+\omega$. Let $D$ be a $\kappa$-dense subset of $\mathbb{R}$ with $|D|=\kappa$, and let

$$
\mathcal{K}=\{(p, q) \times\{d\}: p, q, d \in D \& p<q\} .
$$

For each $f \in \mathfrak{A}$ let

$$
U_{f}=\{\langle x, y\rangle: x \in \mathbb{R} \& f(x)-2<y<f(x)+2\}
$$

and

$$
\mathcal{K}_{f}=\left\{K \in \mathcal{K}:\left|\operatorname{dom}\left(K \cap U_{f}\right)\right|>\kappa\right\} .
$$

Clearly, $\left|\mathcal{K}_{f}\right|=\kappa$. Also, by Lemma 1.8,

the set $A=\bigcup_{f \in \mathfrak{A}} A_{\kappa^{+}}(f)$ has cardinality $\leq \kappa$.

Let $\left\{\left\langle f_{\xi}, K_{\xi}\right\rangle: \xi<\kappa\right\}$ be a transfinite sequence consisting of all pairs $\langle f, K\rangle$ such that $f \in \mathfrak{A}$ and $K \in \mathcal{K}_{f}$. As in Theorem 4.1 we can construct by transfinite 
induction a sequence $\left\{\left\langle x_{\xi}, y_{\xi}\right\rangle: \xi<\kappa\right\}$ such that for every $\xi<\kappa$ the following conditions hold:

(i) $x_{\xi} \notin\left\{x_{\zeta}: \zeta<\xi\right\} \cup A$;

(ii) $\left\langle x_{\xi}, f_{\xi}\left(x_{\xi}\right)+y_{\xi}\right\rangle \in K_{\xi} \cap U_{f_{\xi}}$.

Notice that $y_{\xi} \in(-2,2)$ for every $\xi<\kappa$. Define $g: \mathbb{R} \rightarrow(-2,2)$ by

$$
g(x)= \begin{cases}y_{\xi} & \text { if } x=x_{\xi} \text { for some } \xi<\kappa \\ 0 & \text { otherwise }\end{cases}
$$

Fix an $f \in \mathfrak{A}$. We will prove that $f+g \in \mathcal{U}_{\kappa}$. Let $a<b$ and define $c=(f+g)(a)$ and $d=(f+g)(b)$. Clearly we may assume that $c \neq d$. We will assume that $c<d$, the case $d<c$ being similar.

Let $y \in(c, d) \cap D$. We will find $p, q \in(a, b) \cap D$ such that $p<q$ and

$$
K=(p, q) \times\{y\} \in \mathcal{K}_{f} .
$$

Indeed, if $y \in(-1,1)$, then $[\mathbb{R} \times\{y\}] \subseteq U_{f}$ and the relation (2) holds for any $p, q \in(a, b) \cap D$ with $p<q$. So, suppose that $y \notin(-1,1)$. We will assume that $y \geq 1$, the case $y \leq-1$ being essentially the same.

Then $f(b)+g(b)=d>y \geq 1>f(b)$, so $g(b) \neq 0$. In particular, $b \notin A_{f}^{-}$. So, there exist an $\varepsilon \in(0, d-y)$ and $p, q \in(a, b) \cap D$ such that the set $S=$ $\{x \in(p, q): f(x)>f(b)-\varepsilon\}$ has cardinality greater than $\kappa$. But for every $x \in S$ we have

$$
f(x)+2>f(x)+g(b)>f(b)-\varepsilon+g(b)=d-\varepsilon>y \geq 1>f(x)-2 .
$$

Thus $S \times\{y\} \subseteq U_{f}$ and $(p, q) \times\{y\} \in \mathcal{K}_{f}$.

Let $\xi<\kappa$ be such that $\langle f, K\rangle=\left\langle f_{\xi}, K_{\xi}\right\rangle$. Then $x_{\xi} \in(p, q) \subseteq(a, b)$ and $(f+g)\left(x_{\xi}\right)=f_{\xi}\left(x_{\xi}\right)+y_{\xi}=y$. It follows that $(c, d) \cap D \subseteq(f+g)[(a, b)]$, i.e., that $(f+g)[(a, b)]$ is $\kappa$-dense in $(c, d)$. This completes the proof.

\section{REFERENCES}

1. J. B. Brown, P. D. Humke, and M. Laczkovich, Measurable Darboux functions, Proc. Amer. Math. Soc. 102(3) (1988), 603-612. MR 89b:26003

2. A. M. Bruckner, J. G. Ceder, and M. L. Weiss, Uniform limits of Darboux functions, Colloq. Math. 15(1) (1966), 65-77. MR 33:5794

3. J. G. Ceder, Differentiable roads for real functions, Fund. Math. 65 (1969), 351-358. MR 40:4398

4. J. G. Ceder and T. L. Pearson, Insertion of open functions, Duke Math. J. 35 (1968), 277-288. MR 36:6556

5. K. Ciesielski and A. W. Miller, Cardinal invariants concerning functions, whose sum is almost continuous, Real Anal. Exchange 20(2) (1994-95), 657-672. MR 96h:26003

6. K. Ciesielski and I. Recław, Cardinal invariants concerning extendable and peripherally continuous functions, Real Anal. Exchange 21(2) (1995-96), 459-472. MR 97f:26003

7. U. B. Darji and P. D. Humke, Every bounded function is the sum of three almost continuous bounded functions, Real Anal. Exchange 20(1) (1994-95), 367-369. MR 95m:26004

8. H. W. Ellis, Darboux properties and applications to non-absolutely convergent integrals, Canad. J. Math. 3 (1951), 471-485. MR 13:332d

9. R. G. Gibson and F. Roush, Connectivity functions with a perfect road, Real Anal. Exchange 11(1) (1985-86), 260-264.

10. Z. Grande, A. Maliszewski, and T. Natkaniec, Some problems concerning almost continuous functions, Real Anal. Exchange 20(2) (1994-95), 429-432.

11. A. Maliszewski, Sums of bounded Darboux functions, Real Anal. Exchange 20(2) (1994-95), 673-680. MR 96f:26002 
12. T. Natkaniec, Almost continuity, Real Anal. Exchange 17(2) (1991-92), 462-520. MR 93e:54009

13. - Extendability and almost continuity, Real Anal. Exchange 21(1) (1995-96), 349-355. MR 97g:26002

14. T. Radakovič, Über Darbouxsche und stetige Funktionen, Monatsh. Math. Phys. 38 (1931), $117-122$.

Department of Mathematics, West Virginia University, Morgantown, West Virginia 26506-6310

E-mail address: kcies@wvnvms.wvnet.edu

Department of Mathematics, Pedagogical University, Arciszewskiego 22, 76-200 SŁuPsk, Poland

E-mail address: wspb05@pltumk11.bitnet 